\title{
Assessment and forecasting the spread of SARS- CoV-2 outbreak in Changsha, China: Based on a SEIAR Dynamic Model
}

\section{Wenting Zha}

Hunan Normal University

\section{Nan Zhou}

Hunan Normal University

Guoqun Li

Hunan Normal University

\section{Weitong Li}

Hunan Normal University

\section{Heng Zhang}

Changsha Center for Disease Control and Prevention

\section{Siyu Zhang}

Hunan Centers for Disease Control and Prevention

\author{
Mengxiang Chen \\ Hunan Normal University \\ Ruihua Feng \\ Hunan Normal University \\ Tong Li \\ Hunan Normal University \\ Yuan LV (D ly598598@126.com ) \\ Hunan Normal University
}

\section{Research article}

Keywords: SARS-CoV-2; COVID-19; SEIAR model; Effective regeneration number (Rt); Forecast

Posted Date: March 11th, 2020

DOI: https://doi.org/10.21203/rs.3.rs-16659/v1

License: (c) (1) This work is licensed under a Creative Commons Attribution 4.0 International License.

Read Full License 
1

2

3

4

5

6

7

8

9

10

Title:

Assessment and forecasting the spread of SARS-CoV-2 outbreak in Changsha,

China: Based on a SEIAR Dynamic Model

\section{Author names and affiliations:}

Wen-ting ZHA ${ }^{1}$, Nan ZHOU ${ }^{1}$, Guoqun $\mathrm{LI}^{1}$, Weitong $\mathrm{LI}^{1}$, Heng ZHANG ${ }^{2}$, Siyu ZHANG ${ }^{3}$, Mengxiang $\mathrm{CHEN}^{1}$, Ruihua FENG ${ }^{1}$, Tong $\mathrm{LI}^{1}$, Yuan $\mathrm{LV}^{1^{*}}$

1 Key Laboratory of Molecular Epidemiology of Hunan Province, School of Medicine, Hunan Normal University, Changsha, Hunan, People's Republic of China, 410081.

2 Changsha center for Disease Control and Prevention, Changsha, Hunan, People's Republic of China, 410004.

3 Hunan center for Disease Control and Prevention, Changsha, Hunan, People's Republic of China, 410005.

Wen-ting ZHA and Nan Zhou contributed equally to this work

*Corresponding authors:

Yuan LV

Key Laboratory of Molecular Epidemiology of Hunan Province, School of Medicine, Hunan Normal University, No. 371, Tongzipo Road, Yuelu District, Changsha, HuNan, People's Republic of China

Tel.: +86-15874019359

E-mail: 1y598598@126.com 


\section{ABSTRACT}

Background: A new human coronavirus named SARS-CoV-2 emerged during December 2019 in Wuhan, China. Cases have been exported to other Chinese cities and abroad, which may cause the global outbreak. Chang Sha is the nearest provincial capital city to Wuhan, the first case of COVID-19 in Changsha was diagnosed on January 21, 2020. Estimating the transmissibility and forecasting the trend of the outbreak of SARS-CoV-2 under the prevention and control measures in Changsha could inform evidence based decisions to policy makers.

Methods : Data were collected from the Health Commission of Changsha and Hunan Center for Disease Control and Prevention. A Susceptible-exposed-infections/ asymptomatic- removed (SEIAR) model was established to simulate the transmission of SARS-CoV-2 in Changsha. Berkeley Madonna 8.3.18 were employed for the model simulation and prediction, while the curve fitting problem was solved by the Runge-Kutta fourth-order method, with a tolerance of 0.001.

Results: In this study, we found that Rt was 2.05 from January 21 to 27 and reduced to 0.2 after January 27, 2020 in Changsha. The prediction results showed that when no obvious prevention and control measures were applied, the total number of patients in Changsha would reach the maximum (2.27 million) on the 79th day after the outbreak, and end in about 240 days; When measures have not been fully launched, the total number of patients would reach the maximum (1.60 million) on the 28 th day after the outbreak, and end in about 110 days; When measures have been fully launched, the total number of patients would reach the maximum (234) on the 23rd day after the outbreak, 
and end in about 60 days.

Conclusions: Outbreak of SARS-CoV-2 in Changsha is in a controllable stage under current prevention and control measures, it is predicted that the cumulative patients would reach the maximum of 234 on February 12, and the outbreak would be over on 20 March in Changsha. With the fully implementation of prevention and control measures, it could effectively reduce the peak value, short the time to peak and duration of the outbreak.

Keywords: SARS-CoV-2; COVID-19; SEIAR model; Effective regeneration number (Rt); Forecast

\section{BACKGROUND}

A new human coronavirus, named as severe acute respiratory syndrome coronavirus 2 (SARS-CoV-2) by International Committee on Taxonomy of Viruses on February 11, 2020, emerged during December 2019 in Wuhan, the capital of Hubei province in China [1]. On January 31, 2020, the world health organization (WHO) suggested that there was possible sustained human-to-human transmission of SARS-CoV-2, and announced that this outbreak in China became an Public health emergencies of international concern (PHEIC), which may cause the global outbreak [2]. As of February 17, 2020 (Beijing time), 72528 cases of SARS-CoV-2, which have been named as Coronavirus disease 2019 (COVID-19) have been reported in China, including 1870 deaths, and cases have been detected in at least 25 regions or countries 
outside China, including Japan $(n=520)$, Singapore $(n=77)$, Thailand $(n=35)$, South Korea ( $n=29)$, Malaysia ( $n=22)$ and so on [3]. Data is growing day by day, public health concerns are being paid globally on this outbreak of SARS-CoV-2.

Changsha, the provincial capital of Hunan in China, with more than 8 million residents, which is the nearest provincial capital city to Wuhan (Figure 1). The first case of COVID-19 in Changsha was diagnosed on January 21, 2020, the first level response to major public health emergencies was launched on January 24, and a series of preventive and control measures like isolation of patients, screening of close contacts, media publicity and health education, closure of public places and transportation, prohibiting big parties and visits, personal protection and so on were taken to deal with it. Up to now, the cumulative case of COVID-19 in Changsha was 241, ranking the 7th in cities of China except Hubei province [3].

The genetic features and some clinical findings of the infection in Wuhan have been reported recently [4-7]. It's been a month since the first patient of COVID-19 was reported in Changsha, however, the effect of preventive and control measures, the future epidemic trend in Changsha were unclear. In this study, we analysed the epidemiological characteristics of patients, developed a Susceptible-exposed-infections/asymptomaticremoved (SEIAR) dynamic model to estimate the transmissibility and dynamic of the transmission of the virus, evaluate the effect of the prevention and control measures, and forecast the trend of the epidemic situation of SARS-CoV-2 in Changsha, in order to inform evidence based decisions to health decision and policy makers. 


\section{METHODS}

\section{Data source}

The reported case of COVID-19 from January 21 to February 17, 2020 in Changsha were collected from the Health Commission of Changsha [8]. The proportion of recessive infection in Hunan were collected from the Hunan Center for Disease Control and Prevention. The onset date of the first case was on January 21, 2020, and the epidemic curve from January 21 to February 17, 2020 was collected for our study, the simulation time step was one day.

\section{Models and statistical analysis}

\section{SEIAR model}

A SEIAR model was established to simulate the transmission of SARS-CoV-2 in Changsha. Population in this model was divided into five categories according to the disease status (Figure 2): susceptible (S), exposed (E), infected (I), asymptomatic (A) and recovered (R). The model was developed based on the following facts or assumptions, which assumed that some individuals moved among categories because of infection or recovery: 1) The population was defined as closed and stable; 2) Susceptible person (S) was assumed to have an equal infected rate $(\beta)$ with the symptomatic infected person (I) and $\kappa \beta$ with asymptomatic infected person (A); 3) After infected, the exposed person (E) would turn to I or A after a certain exposed period $(1 / \omega)$, the number of newly I and A per unit time was $\omega \mathrm{E}$; 4) $\gamma$ meant the removal rate, the number of newly recovered individuals $(R)$ per unit time was $\left(\gamma_{1} \mathrm{~A}+\gamma_{2} \mathrm{I}\right)$; 5) The fatality rate were 
ignored, because it was very low in Changsha.

The corresponding model equations were as follows, $d \mathrm{~S} / d \mathrm{t}, d \mathrm{E} / d \mathrm{t}, d \mathrm{t} / d \mathrm{t}, d \mathrm{~A} / d \mathrm{t}$ and $d \mathrm{R} / d \mathrm{t}$ denoted the number of individuals (n) at time $\mathrm{t}$ in the corresponding categories:

$$
\left\{\begin{array}{l}
d S / d t=-\beta S I-\kappa \beta S A \\
d E / d t=\beta S I+\kappa \beta S A-p w E-(1-p) w E \\
d A / d t=p w E-\gamma_{1} A \\
d I / d t=(1-p) w E-\gamma_{2} I \\
d R / d t=\gamma_{1} A+\gamma_{2} I
\end{array}\right.
$$

The effective regeneration number (Rt) was calculated by the following formula according to the model:

$$
R t=\beta S_{t}\left(\frac{1-p}{\gamma_{2}}+\frac{\kappa p}{\gamma_{1}}\right)
$$

\section{Parameter estimation}

There were six parameters in all models in this study, which were infection rate $(\beta)$, the ratio of transmission probability of A to $\mathrm{I}(\kappa)$, the proportion of asymptomatic infection rate of people $(P)$, latency coefficient $(\omega)$, removal rate of A $\left(\gamma_{1}\right)$ and removal rate of I $\left(\gamma_{2}\right)$, all parameters and initial values of each categorie were list in Table 1.

a) As of February 12, 2020, 972 cases with COVID-19 and 121 cases with asymptomatic infection were reported in Hunan province, Changsha is the provincial capital of Hunan, so the the proportion of asymptomatic infection rate of people in our model was $P=121 /(972+121)=11 \%$.

b) The mean incubation period was 5.2 days (95\% confidence interval [CI]: $4.1-7.0)$ [4], we set the same value (5.2 days) of the incubation period in our study, so, 
$\omega=1 / 5.2$.

132

c) $\gamma$ meant the removal rate, $1 / \gamma$ meant the infective period. While once symptomatic infected person was diagnosed, they would be isolated, the $1 / \gamma_{2}$ represented the time from onset to diagnosis of symptomatic infected person. From the actual data, we have calculated that the average time from onset to diagnosis was 3 days, so, $\gamma_{2}=1 / 3$.

d) While those asymptomatic infected person would not be easily found and isolated, the recovery day was equal to 14 days [9], so, the infective period of asymptomatic infection was $\gamma_{1}=1 / 14$.

\section{Simulation methods}

Considering the implementation degree of the prevention and control measures, we divided the time into two periods based on the measures in Changsha. The first period was from January 21 to 27, 2020, when the prevention and control measures have not been fully launched, the second period was from January 28 to February 17, 2020, when the prevention and control measures have been fully launched.

We fitted the data from the outbreak of SARS-CoV-2 in Changsha to a SEIAR model curve to estimate $\beta$ and $\kappa$ in these two periods respectively. Berkeley Madonna 8.3.18 and Microsoft Office Excel 2010 software were employed for the model simulation and data management, respectively. Graphpad prism 5 was used for the figure development, while the curve fitting problem was solved by the Runge-Kutta fourth-order method, with a tolerance of 0.001 . A Goodness of fit test ( $\chi^{2}$ test) was performed using the IBM-SPSS software, in which the significance level was $\alpha=0.05$.

\section{Forecast}



three models were compared.

a) The first prediction model was to forecast the epidemic situation in Changsha based on the parameters in the early stage of Wuhan $\left(\mathrm{R}_{0}=2.68\right)[10]$, that was, the parameters when almost no prevention andcontrol measures were taken.

b) The second prediction model was to forecast the epidemic situation in Changsha according to the parameters obtained by model fitting from January 21 to 27, 2020, when the prevention and control measures have not been fully launched.

c) The third prediction model was to forecast the epidemic situation in Changsha according to the parameters obtained by model fitting from January 28 to February 17, 2020, when the prevention and control measures have been fully launched.

\section{RESULTS}

\section{Epidemiological features of the outbreak of SARS-CoV-2 in Changsha}

From January 21 to February 17 in the year 2020, 241 patients of COVID-19 in Changsha were reported. The sex ratio of men to women was 120:121, the oldest and youngest were 84 and 3 years old, respectively, the median age was 45 years old. 40 people were from Wuhan or other areas in Hubei, accounting for $16.6 \%$. The median time of patients from onset to diagnosis in Changsha was 3 days, and 217 patients just had mild symptoms at the time of diagnosis, which accounting for $90.0 \%$. Among the eight districts under the jurisdiction of Changsha, Furong, Kafu and Yulu district reported the largest number of patients, a total of 166 cases, which accounting for $68.9 \%$. 
As of February 17, 2020, 92 patients were discharged from hospital, 2 died, 147 patients were in hospital now, 10 of them were serious. The distribution of time among patients was shown in Figure 3.

\section{The effect of prevention and control measures in Changsha at different time}

We divided the time into two periods based on the prevention and control measures in Changsha, which was from January 21 to 27, and from January 28 to February 17, 2020. The results of curve fitting of the outbreak data in Changsha and SEIAR model showed that the simulated result agreed well with the reported data when $\beta_{1}$ equal to $5.02 \times 10^{-7}, \mathrm{R}_{1}$ equal to $2.05, \kappa_{1}$ equal to 0.93 from January 21 to 27,2020 , and $\beta_{2}$ equal to $6.00 \times 10^{-8}, \mathrm{R}_{2}$ equal to $0.20, \kappa_{2}$ equal to 0.95 from January 27 to February 17 , $2020\left(\mathrm{R}^{2}=0.97, P<0.001\right)$. The model fitting diagram was shown in Figure 4.

\section{Forecast of the outbreak of SARS-CoV-2 in Changsha}

The prediction results showed that when no obvious measures were applied, the total number of patients in Changsha would reach the maximum on the 79th day after the outbreak, which was 2.27 million patients, and the outbreak would end in about 240 days; When prevention and control measures have not been fully launched, the total number of patients in Changsha would reach the maximum on the 28th day after the outbreak, which was 1.60 million patients, and the outbreak would end in about 110 days; When prevention and control measures have been fully launched, the total number of patients in Changsha would reach the maximum on the 16th day after the fully intervention, that is, on the 23rd day after the outbreak (February 12, 2020), which was 234 patients, and the outbreak would end in about 60 days (March 20, 2020). The 
forecast results were shown in Figure 5.

\section{DISCUSSION}

In this study, we found that the number of male patients was similar to female, which was different from the patients in Wuhan [5,6], but the same with the results of the whole country $[11,12]$, which may be due to the different study sites and the number of cases, further studies are needed to find out whether there are differences between men and women. People of all ages were generally susceptible to SARS-CoV-2, and the median age of patients were 45 years old, which is consistent with the results of other provinces in China [11,12]. 16.6\% patients were from Wuhan or other areas in Hubei, $90.0 \%$ patients just had mild symptoms at the time of diagnosis. Since the sealed off Wuhan on January 23, there have been few, almost none people from Wuhan to Changsha, so, some early patients may have come from or contact with Wuhan or other areas in Hubei, but the latter patients were mostly local transmission patients. Since the first case of COVID-19 in Changsha was diagnosed on January 21, 2020, a series of preventive and control measures were taken to deal with it, most of the patients were found and diagnosed at an earlier time, which would reduce the proportion of severe patients and reduce the harm of this disease.

The proportion of asymptomatic infection of SARS-CoV-2 was low, just accounting for $11.1 \%$, which was higher than Severe acute respiratory syndrome (SARS) and Middle east respiratory syndrome (MERS) [13,14], but lower than influenza [15]. The probability of transmission in asymptomatic infected patients were not very different 
222

223

224

225

226

227

228

229

230

231

232

from that of symptomatic infected patients ( $\kappa=0.93$ to 0.95$)$, which is what we need to pay attention to. So, in the later stage, we should carry out nucleic acid testing to find out more asymptomatic infected people, especially from the key groups, such as close contacters of patients, medical workers, employees returning to enterprise and students returning to school from Wuhan or other areas of Hubei, to reduce the impact of asymptomatic infection on the re-outbreak or epidemic of SARS-CoV-2.

To control the transmission of virus, it is important to decrease $\mathrm{R}_{\mathrm{t}}$. Due to the different research places and time, the $\mathrm{R}_{0}$ or $\mathrm{R}_{\mathrm{t}}$ obtained by scholars were quite different, which ranged from 1.10 to 6.47 in China [16-19]. The number of patients is changing rapidly, the measures taken in different places and time are not all the same, so, it is necessary to adjust the model and parameters according to different situations of different regions. In this study, we found that Rt was 2.05 from January 21 to 27 in Changsha, which was lower than the $\mathrm{R}_{0}$ in Wuhan at the early time, and Rt reduced to 0.2 , lower than 1 after January 27, 2020, which meant that the SARS-CoV-2 in Changsha was in a controllable stage under current prevention and control measures.

The prediction results of the model showed that the cumulative patients of COVID-19 in Changsha would reach the maximum value of 234 on February 12, which was fitting well to the actual situation, and the outbreak of SARS-CoV-2 in Changsha would end around March 20. Compared with no or initial measures, with the fully implementation of the epidemic prevention and control measures in Changsha, it could effectively reduce the peak value, short the time to peak and duration of the outbreak, reduce the harm brought by the epidemic. Scientific and effective prevention and control 
provides a reference for dealing with new infectious diseases in the future. the simulation and the actual outbreak, which is the limitation in our study. After February 17, the resumption of work in enterprises and schools may have some impact on the development of the epidemic, so the model and parameters need to be adjusted according to the actual situation.

\section{CONCLUSIONS}

The proportion of asymptomatic infections of SARS-CoV-2 in Changsha was low, but the probability of transmission was not very different from that of patients; Outbreak of SARS-CoV-2 in Changsha is in a controllable stage under current prevention and control measures, it is predicted that the cumulative patients would reach the maximum of 234 on February 12, and the outbreak would be over on March 20 in Changsha. With the fully implementation of prevention and control measures, it could effectively reduce the peak value, short the time to peak and duration of the outbreak.

\section{List of abbreviations}

1. Severe acute respiratory syndrome coronavirus 2 (SARS-CoV-2)

2. World health organization (WHO)

3. Public health emergencies of international concern (PHEIC)

4. Coronavirus disease 2019 (COVID-19) 
6. Regeneration number (Rt)

267

7. Confidence interval $(C I)$

268

8. Severe acute respiratory syndrome (SARS)

269

9. Middle east respiratory syndrome (MERS)

270

Declarations

272

Ethics approval and consent to participate

273

Not applicable

274

275

Consent for publication

276

Not applicable

277

Availability of data and materials

279

All data and material in our study were availability

280

The reported case of COVID-19 in Changsha were collected from the Health

281

Commission of Changsha. http://wsjkw. changsha.gov.cn/ztzl_1/fkxxgzbd/index.html.

282

The proportion of recessive infection in Hunan were collected from the Hunan Center for Disease Control and Prevention.

\section{Competing interests}

The authors declare that they have no competing interests. 


\section{Funding}

289

290

291

292

293

294

295

296

297

298

299

300

301

302

303

304

305

306

307

308

309

The work was supported by Education Project of Hunan Provincial Department [2017] No. 451, [2017] No. 452 and JG2018B041.

The funder had in our study was the correspondence author (Yuan LV).

\section{Authors' contributions}

$\mathrm{W} \mathrm{Z}$ and $\mathrm{N} \mathrm{Z}$ mainly responsible for the data analysis, model building and drafting of article;

G L and W L mainly responsible for the chart making;

$\mathrm{H} \mathrm{Z}, \mathrm{S} \mathrm{Z}, \mathrm{M} \mathrm{C}$ and R F mainly responsible for the collection of data and searching for relevant parameters;

T L mainly responsible for the correction of English;

Y L mainly responsible for sponsorship of funds and final review of the article.

All authors read and approved the final manuscript.

\section{Acknowledgements}

We are grateful to everyone who helped us to complete this study successfully.

\section{REFERENCES}

[1]World Health Organization. Coronavirus. World Health Organization, cited January 19, 2020. Available:309. https://www.who.int/health-topics/coronavir.

[2]World Health Organizaiton. Statement on the second meeting of the International 
HealthRegulations Emergency Committee regarding the outbreak of novel coronavirus (2019-nCoV). https://www.who.int/news-room/detail/30-01-2020 statement on the second meeting of the international health regulations.

[3]Chinese Center for Disease Control and Prevention. Distribution of COVID-19. http://2019ncov.chinacdc.cn/2019-nCoV.

[4]Qun Li, M. Xuhua Guan, Peng Wu,et al. Early Transmission Dynamics in Wuhan, China, of Novel Coronavirus-Infected Pneumonia. N Engl J Med, 2020; published online Jan 24. http://doi:10.1056/NEJMoa2001316.

[5]Huang C, Wang Y, Li X, et al. Clinical features of patients infected with 2019 novel 315 coronavirus in Wuhan, China. Lancet, 2020; http://doi: 10.1016/S0140-6736(20) $30183-5$

[6]Nanshan Chen, Min Zhou, Cuan Dong, et al. Epidemiological and clinical characteristics of 99 cases of 2019 novel coronavirus pneumonia in Wuhan, China: a descriptives study. Lancet 2020; 395: 507-13. https://doi.org/10.1016/S0140-6736(20) $30211-7$.

[7]Zhu N, Zhang D, Wang W, et al. A Novel Coronavirus from Patients with 320 Pneumonia in China, 2019. N Engl J Med, 2020; http://doi: 10.1056/NEJMoa2001017. [8]The Health Commission of Changsha. The notification of COVID-19. http://wsjkw. changsha.gov.cn/ztzl_1/fkxxgzbd/index.html.

[9]Li Liming, Liang Xiaofeng, Jiang Qingwu, et al. Latest understanding of the epidemiology of COVID-19. Chinese Journal of epidemiology, 2020; 41 (2): 139-144. [10]Joseph T Wu, Kathy Leung, Gabriel M Leung. Nowcasting and forecasting the 
potential domestic and international spread of the $2019-\mathrm{nCoV}$ outbreak originating in Wuhan, China: a modelling study. Lancet, 2020, published online January 31. http://doi.org/10.1016/S0140-6736(20)30260-9.

[11]Wei-jie Guan, Zheng-yi Ni, Yu Hu, al et. Clinical characteristics of 2019 novel coronavirus infection in China, Preprint in MedRXiv, 2020. https://doi.org/10.1101 $/ 2020.02 .06 .20020974$.

[12]China Center for Disease Control and prevention. Epidemiological characteristics of covid-19. Chinese Journal of epidemiology, 2020; 41 (2): 145-151.

[13]Wilder-Smith A, Teleman MD, Heng BH, Earnest A, Ling AE, Leo YS.

Asymptomatic SARS coronavirus infection among healthcare workers,

Singapore. Emerg Infect Dis. 2005;11(7):1142-1145. http://doi:10.3201/eid1107.041165

[14]Kin on Kwok, Arthur Tang, Vivian wei, et al. Epidemic models of contact tracting:

Systematic review of thansmission studies of severe acute respiratory syndrome and middle east respiratory syndrome. Computational and structural biotechnology journal, 2019; 17:186-194. http://doi.org/10.1016/j.csbj.2019.01.003.

[15]Wenti Xu, Tianmu Chen, Xiaochun Dong, et al. Outbreak detection and evaluation of a school-based influenza-like-illness syndromic surveillance in Tianjin, China. Plos one, 2017; 8:1-10. https://doi.org/10.1371/journal. pone.0184527.

[16]Isaac I. Bogoch, Alexander Watts, Andrea Thomas-Bachli, et al. Pneumonia of unknown aetiology in Wuhan, China: potential for international spread via commercial airtravel. Journal of Travel Medicine, 2020; 1-3. http://doi: 10.1093/jtm/taaa008. 
[17]Jonathan M. Read, Jessica R.E. Bridgen, Derek A.T. Cummings, Antonia Ho, Chris P.

Jewell. Novel coronavirus NCP: early estimation of epidemiological parameters and epidemic predictions. MedRxiv preprint posted online, Jan 27, 2020.https://doi.org/10. $1101 / 2020.01 .23 .20018549$

[18]Tianmu Chen, Jia Rui, Qiupeng Wang, et al. A mathematical model for simulating the transmission of Wuhan novel Coronavirus. BioRxiv preprint posted online, Jan 19, 2020. http://doi: 10.1101/2020.01.19.91166.

[19]Shi Zhao, Salihu S. Musa, Qianying Lin, et al. Estimating the Unreported Number of Novel Coronavirus (2019-nCoV) Cases in China in the First Half of January 2020: A Data-Driven Modelling Analysis of the Early Outbreak. Journal of clinical medicine, 2020; 9, 388. http://doi:10.3390/jcm9020388.

\section{Tables and Figures}

Table 1 List of parameters and initial values of each categorie in model

Figure 1 The geographical location map of Changsha

Figure 2 Flow chart of SEIAR model of SARS-CoV-2

Figure 3 The distribution of time among patients in outbreak of SARS-CoV-2 in Changsha

Figure 4 The result of curve fitting of actual data and SEIAR model

Figure 5 The forecast results with different parameters 
Figures

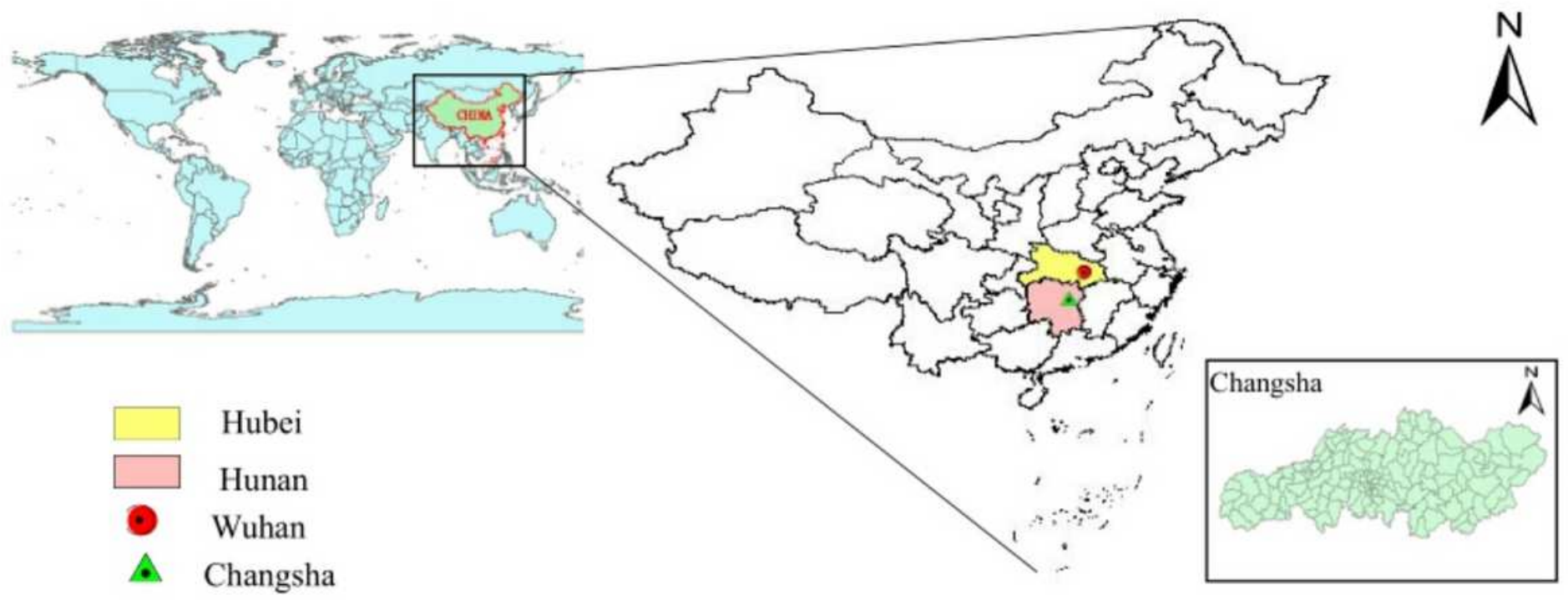

Figure 1. Geographical location map of Changsha

\section{Figure 1}

The geographical location map of Changsha. Note: The designations employed and the presentation of the material on this map do not imply the expression of any opinion whatsoever on the part of Research Square concerning the legal status of any country, territory, city or area or of its authorities, or concerning the delimitation of its frontiers or boundaries. This map has been provided by the authors.

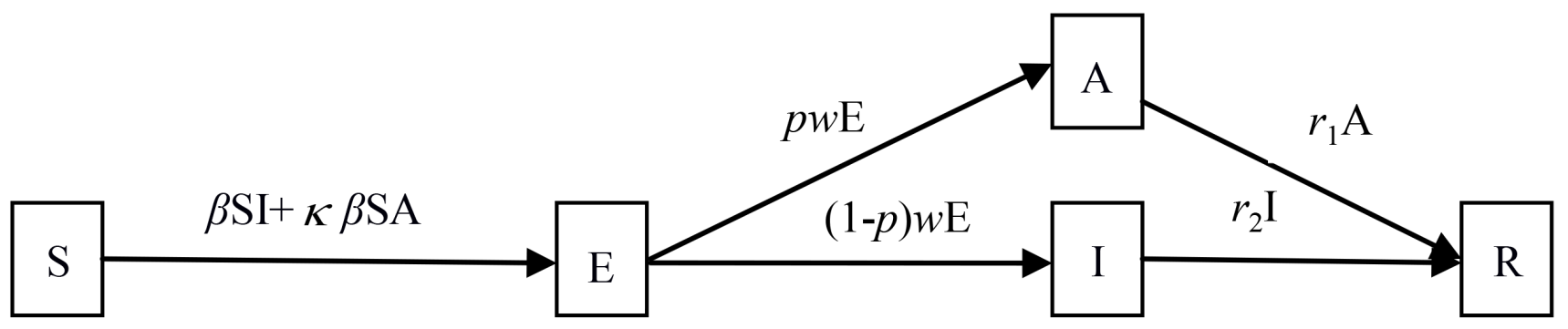

Figure 2 Flow chart of SEIAR model of SARS-CoV-2

Figure 2

Flow chart of SEIAR model of SARS-CoV-2 


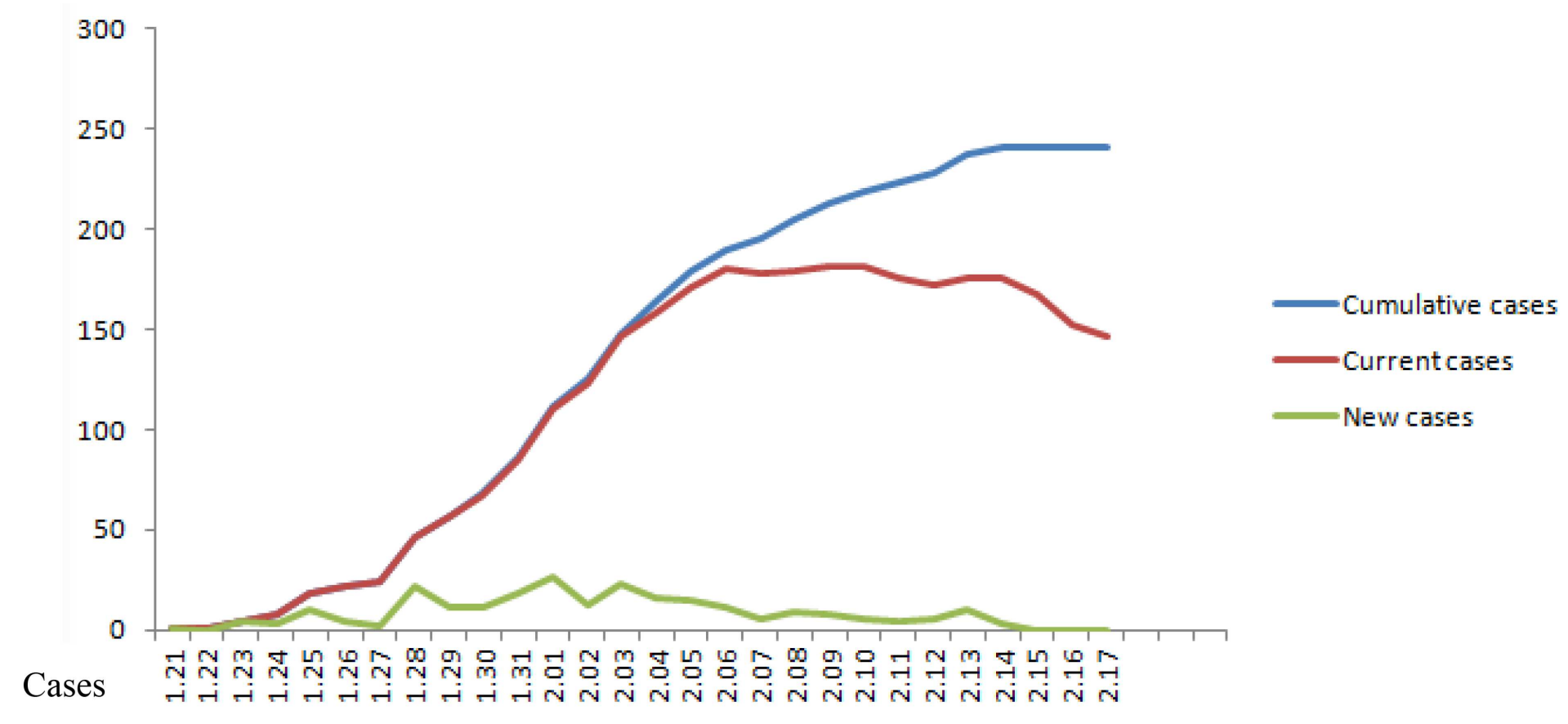

Time

Figure 3 The distribution of time among patients in outbreak of SARS-CoV-2 in Changsha

\section{Figure 3}

The distribution of time among patients in outbreak of SARS-CoV-2 in Changsha

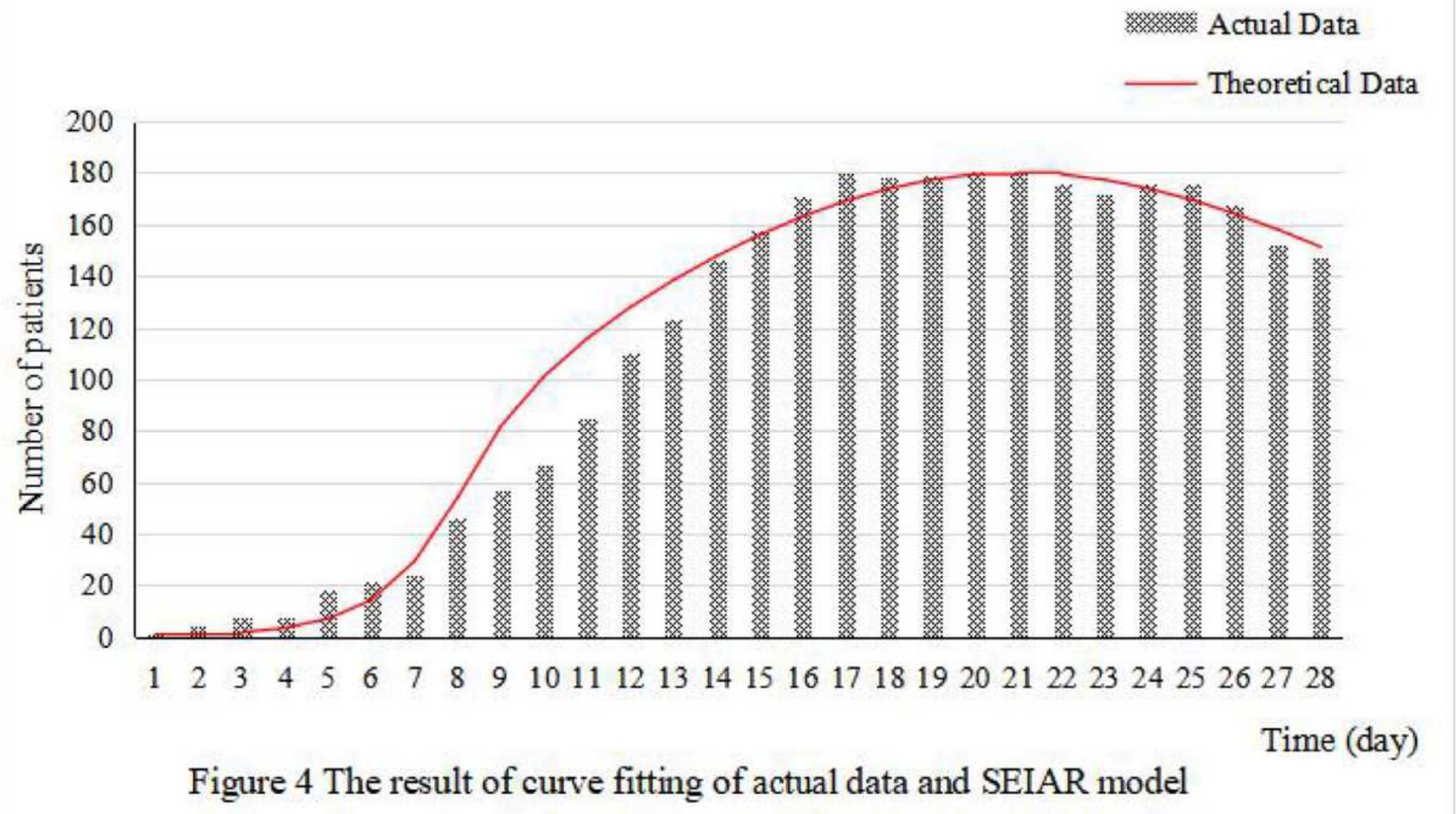


Figure 4

The result of curve fitting of actual data and SEIAR model

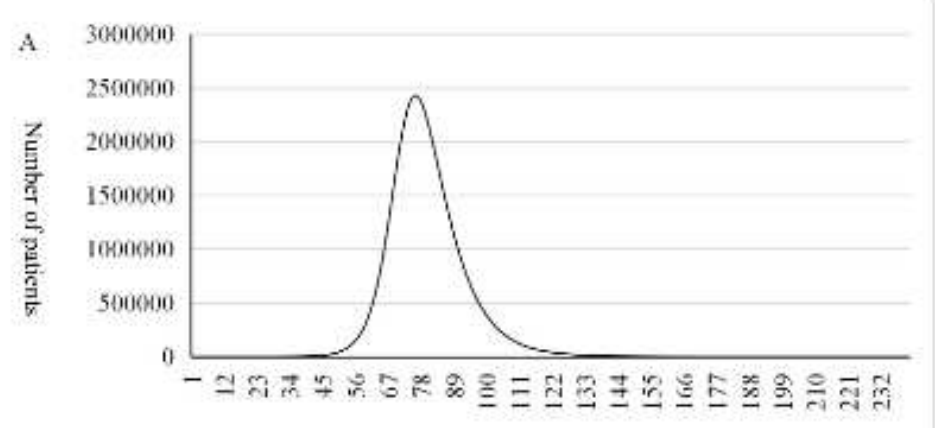

Time (day)
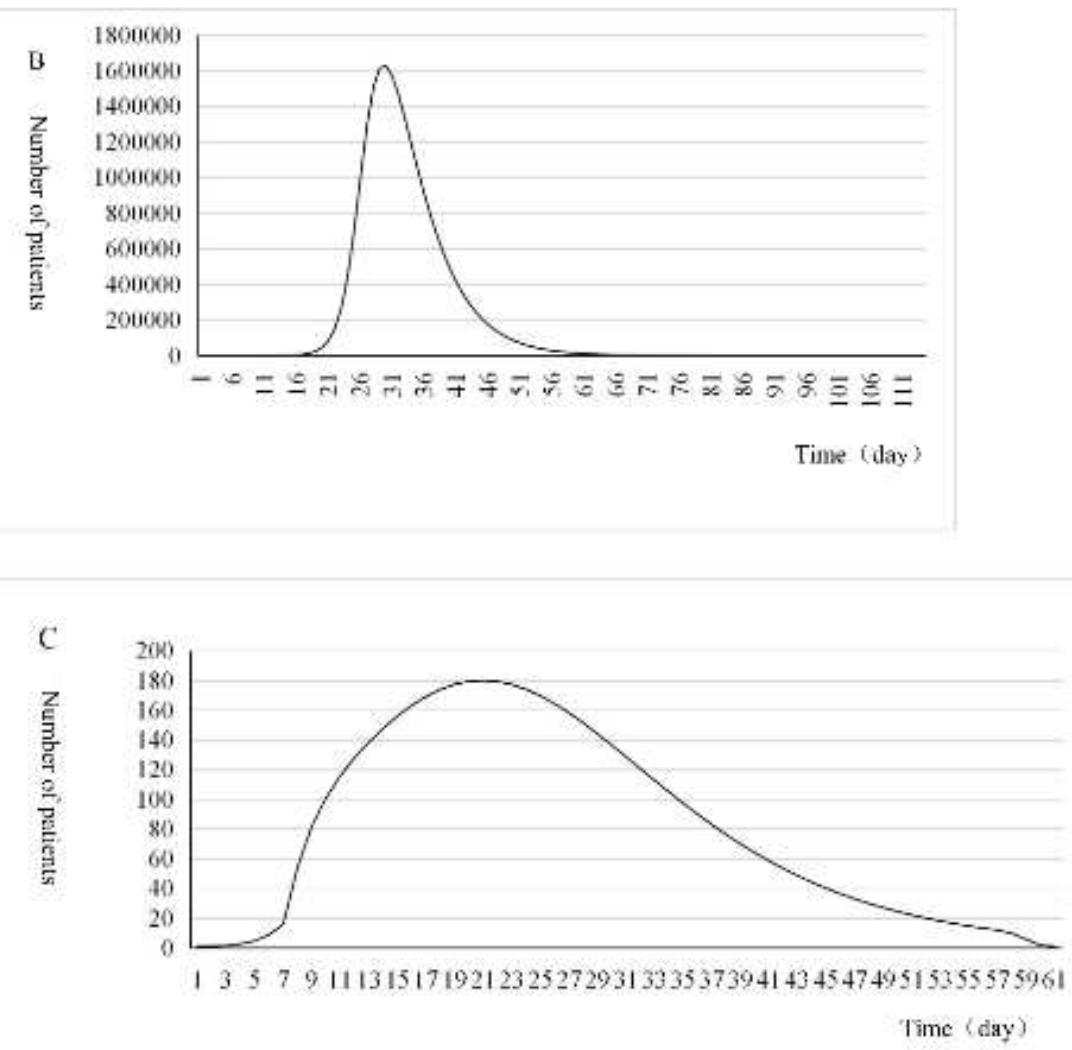

Nove: $5 \mathrm{~A}$ the forecas. Yesults wit parameers in the early stage or Whan, when almos no poevention and control messures were taken; $5 \mathrm{~B}$ Tbe forecast resuls with paramescrs obeniacd by model firting from

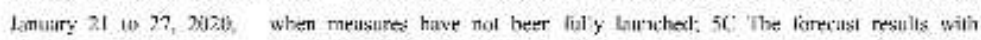

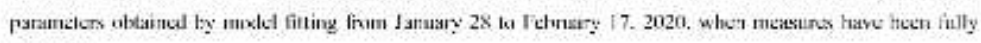
taunctiod.

Figure 5 The forecast results with different parameters

\section{Figure 5}

The forecast results with different parameters 


\section{Supplementary Files}

This is a list of supplementary files associated with this preprint. Click to download.

- Table1.pdf 